\title{
Prolonged Disease Stabilization and
} Tolerability in a Nuclear Protein in Testis Midline Carcinoma Patient Treated with Dual Histone Deacetylase and Phosphoinositide 3-Kinase Inhibitor CUDC-907

\author{
Pamela Munster', Nilson Wu', Meaghan McMahon², Robert Gharavi², David Tuck ${ }^{2}$ \\ ${ }^{1}$ Division of Hematology and Oncology, University of California, San Francisco, USA \\ ${ }^{2}$ Curis, Inc., Lexington, Massachusetts, USA \\ Email: robert.gharavi@gmail.com
}

How to cite this paper: Munster, $\mathrm{P}$., $\mathrm{Wu}$, N., McMahon, M., Gharavi, R. and Tuck, D. (2018) Prolonged Disease Stabilization and Tolerability in a Nuclear Protein in Testis Midline Carcinoma Patient Treated with Dual Histone Deacetylase and Phosphoinositide 3-Kinase Inhibitor CUDC-907. Case Reports in Clinical Medicine, 7, 451-460.

https://doi.org/10.4236/crcm.2018.77039

Received: July 2, 2018

Accepted: July 21, 2018

Published: July 24, 2018

Copyright $\odot 2018$ by authors and Scientific Research Publishing Inc. This work is licensed under the Creative Commons Attribution International License (CC BY 4.0).

http://creativecommons.org/licenses/by/4.0/

\section{c) (†) Open Access}

\begin{abstract}
Introduction: Nuclear protein in testis midline carcinoma (NMC) is a rare and extremely aggressive carcinoma (median survival $<7$ months) with no effective treatment options. CUDC-907 is a novel small molecule inhibitor of histone deacetylase (HDAC) and phosphoinositide 3-kinase (PI3K) enzymes currently being investigated in multiple tumor types, including NMC. Case Report: A 61-year old female NMC patient enrolled on study CUDC-907-102 (NCT02307240) after rapidly progressing through two prior treatments. The patient's assessable sites of disease consisted of right pleural effusion, right hilar soft tissue, and segment IV liver. Treatment was well tolerated with toxicities primarily consisting of manageable diarrhea and thrombocytopenia. The patient remains on active treatment after more than 32 months of stable disease. Discussion: Dysregulation of MYC in NMC is believed to play a central role in pathogenesis. CUDC-907 has demonstrated potent suppression of MYC expression and anti-tumor activity in preclinical NMC models, providing a mechanistic rationale for the prolonged disease stabilization observed here. The treatment of additional NMC patients with CUDC-907 is needed to further evaluate this promising report. Conclusion: This case demonstrates a rare success in the treatment of a devastating disease using only a novel small molecule, warranting further investigation of CUDC-907 in NMC.
\end{abstract}

\section{Keywords}

NMC, MYC, CUDC-907, HDAC, PI3K 


\section{Introduction}

Nuclear protein in testis (NUT) midline carcinoma (NMC) is a genetically defined, rarely diagnosed, and poorly differentiated type of squamous cell carcinoma [1] [2] [3] [4]. Initially described in children and young adults, NMC has since been reported in patients of all age groups. Due to limitations on comprehensive cytogenetic and molecular assessments of adult carcinomas, accurate estimates on the actual prevalence and incidence of NMC are unavailable, and the current estimated numbers are likely to be highly underreported [1]. With the introduction of a diagnostic immunohistochemistry assay in 2009 and the establishment of an international NMC registry (http://www.nmcregistry.org/) in 2010, more patients will present with diagnosed NMC [1] [5]. The disease is extremely aggressive and invariably fatal despite aggressive chemotherapy and radiation treatments. With a reported median overall survival (OS) of less than 7 months, NMC represents a rapidly deadly disease with an urgent need for new effective treatment options [1] [3] [6].

The pathogenesis of NMC is genetically defined by the rearrangement and translocation of NUT most often with either the bromodomain-containing protein 4 (BRD4; [t(15;19) (q14;p13.1)]) or BRD3 ( $\mathrm{t}(15 ; 9)$ (q14;p34.2)). The function of NUT is poorly understood and its expression is usually restricted to the germ cells of the testis and ovaries [5]. BRD4 and BRD3 belong to the bromodomain and extra-terminal domain family (BET) of proteins which bind to acetylated lysine residues on histone proteins and function as epigenetic readers and regulators of transcriptional activity [7]. Both proteins are believed to be essential in the transcription of oncogenes such as c-MYC, MYCN, BCL2, and FOSL1 [8]. In approximately $70 \%$ of cases, NMC is caused by the fusion of NUT (chromosome 15) with BRD4 (chromosome 19), forming the BRD4-NUT oncogene [9]. In the remaining cases, $N U T$ is fused with $B R D 3$ (25\%) or to unidentified genes (5\%). In recent reports, a NSD3-(nuclear receptor binding SET domain 3) NUT fusion was also identified, and a novel three-way translocation involving chromosomes 9, 15, and 19 [10] [11] [12]. The BRD-NUT fusion proteins appear to contribute to carcinogenesis by interacting with chromatin, triggering aberrant histone acetylation, and blocking cellular differentiation. In vitro studies have demonstrated that MYC is a key downstream target of BRD4-NUT, which associates with the $M Y C$ promoter and is required for MYC expression. The dysregulation of $M Y C$ by BRD-NUT fusion proteins is believed to play a central role in the pathogenesis of NMC [13] [14].

CUDC-907 is a novel oral small molecule that potently inhibits class 1 phosphoinositide 3-kinase (PI3K; $\alpha, \beta$, and $\delta$ isoforms) as well as histone deacetylase (HDAC; class I and II) enzymes, blocking cellular proliferation and inducing apoptosis in a variety of cancer cell lines. Preclinical experiments have demonstrated that CUDC-907 dramatically decreased MYC mRNA and protein levels in multiple MYC-dependent cell and animal models. Furthermore, CUDC-907 has been shown to effectively inhibit the growth of human NMC cell lines in 
culture, potently and rapidly eliminate MYC protein levels in these cells, and is active in in vivo xenograft models of human NMC (BRD4-NUT fusion) [15]. The proposed mechanisms for the observed CUDC-907 induced MYC suppression include reduced $M Y C$ expression at the transcriptional level via HDAC inhibition and reduced MYC protein stability at the post-translational level via PI3K inhibition [15].

CUDC-907 is currently being investigated in 3 clinical trials; a Phase 1 trial of CUDC-907 with and without rituximab in relapsed/refractory lymphoma and multiple myeloma patients, a phase 2 study of CUDC-907 in relapsed/refractory diffuse large B-cell lymphoma patients, with a primary analysis population of MYC-altered patients, and a Phase 1 study of CUDC-907 in patients with solid tumors, including NMC patients. Herein we report on the case of a 61 year old female NMC patient that enrolled on the Phase 1 CUDC-907-102 (NCT02307240) solid tumor study in November 2015 and remains on active treatment. The patient consented to the publication of this case report.

\section{Case Report}

In January 2015 the patient, who is a never smoker, reported an approximate 8 -week history of non-productive cough and wheezing. A chest x-ray and follow-up computed tomography (CT) scan showed a 9-cm right upper lobe mass with compression of superior vena cava and mediastinal extension versus right paratracheal/pretracheal lymph node involvement and repeated pleural effusions. Routine hematoxylin and eosin stained sections showed a high-grade malignant neoplasm eliciting a mixed desmoplastic and inflammatory response. Interpretation was hampered by the small size of the specimen and "crush" artifact, considered possibly inherent to the neoplasm. The only immunohistochemical (IHC) marker that was convincingly staining positive was p63, usually seen with squamous carcinomas. However, other epithelial markers (AE1/AE3, CAM5.2, CK5/6, CK7 and CK20) were negative. Hematopoietic markers including CD45, CD2, CD3, CD20, CD30 and ALK were negative as were a melanocytic marker (S-100) and neuroendocrine markers (TTF-1, CD56 and chromogranin). The case was referred for a consultative opinion and definitive diagnosis. In February 2015 a histologic section of the original right upper lobe lung biopsy showed several extensively crushed foci of atypical, somewhat dyshesive larger cells in clusters and linear arrangements, some with vasculocentricity. In some areas the cells appeared to show ovoid nuclei, open chromatin, inconspicuous nucleoli, and moderate amount of cleared cytoplasm. Further IHC staining performed showed that the atypical cells did not express synaptophysin, kappa or lambda immunoglobulin light chains RNA, MPO, HHV8, CD33, SOX-10, ERG, desmin or CD99. IHC and fluorescent in situ hybridization testing of tumor cells confirmed the diagnosis of NMC and the presence of BRD4-NUT translocations $\mathrm{t}(15 \mathrm{q} 14 ; 19 \mathrm{p} 13.1)$.

The patient's first reported treatment was with carboplatin and paclitaxel with 
adjuvant radiotherapy to the lungs from 25 February 2015 until disease progression on 11 May 2015 (best response of stable disease). The patient's second-line of treatment was with the investigational BET inhibitor GSK525762 from 11 June 2015 to 21 September 2015 (best response of stable disease). Other than a dose reduction due to thrombocytopenia, the patient reportedly tolerated treatment well with mild nausea, vomiting, decreased appetite, and approximately 10 pounds of weight loss and an increasing need for thoracentesis to remove pleural fluid. A later CT scan showed progressive disease by RECIST on 30 September 2015.

In October 2015 the patient was referred to the University of California San Francisco for management of their metastatic NMC and consideration for enrollment on the CUDC-907-102 clinical trial. A CT scan of the chest, abdomen, and pelvis demonstrated evidence of disease progression with pulmonary nodularity, increasing mediastinal adenopathy and an increased right pleural effusion. During screening for participation on the CUDC-907-102 trial the patient underwent thoracentesis on 28 October 2015, which was reported to significantly improve their pulmonary symptoms. A history of asthma (2012 - ongoing), type II diabetes mellitus (2016 - ongoing), hypertension (1977 - ongoing), intermittent tachycardia (August 2015 - ongoing), non-productive cough (September 2014 - ongoing), and right pleural effusion (October 2015 - ongoing) were all reported. ECOG performance status of 1 was noted at screening and remained so throughout study participation. The patient entered the trial without any target lesions present. An ill-defined thoracic mass, right pleural effusion, segment IV liver, and right hilar soft tissue were selected as non-target lesions to follow for response assessments.

On 05 November 2015, the patient began CUDC-907 treatment at the recommended phase 2 dose of $60 \mathrm{mg}$ daily for 5 days on, 2 days off (5/2) on continuous 21-day cycles. On the first day of treatment Grade 2 diarrhea was reported which continued for 14 days. Also on the same day, Grade 1 nausea was reported which became Grade 2 the following day and continued for 3 days. On the second day of study treatment loperamide and ondansetron were initiated to help control the symptoms of diarrhea and nausea, respectively. On the $12^{\text {th }}$ day of treatment, Grade 1 hypokalemia and Grade 2 thrombocytopenia were reported. Concomitant treatment with potassium chloride and lomotil (replacing loperamide) were also initiated on this day. On the $15^{\text {th }}$ day of Cycle 1 treatment was held for the rest of the cycle (7 days) to alleviate the symptoms of thrombocytopenia and diarrhea. During this final week of Cycle 1, a Grade 1 maculo-papular rash possibly related to study treatment was also reported and persisted for over 2 months. The last 4 scheduled doses of Cycle 2 were held due to Grade 3 thrombocytopenia and Cycle 3 was initiated at the first dose reduction level of $60 \mathrm{mg} 4$ days on, 3 days off (4/3). During Cycle 5, intermittent Grade 1 thrombocytopenia worsened to Grade 3. Treatment was then held for 5 days and a second dose reduction occurred per protocol to $30 \mathrm{mg} \mathrm{5/2}$ at the start of Cycle 6 . 
Initial staging studies after cycle 2 showed decreased right pleural effusion and decreased extent of right lower lobe peribronchial opacities and right major fissure nodular opacities with improved aeration of the right lower lobe. Restaging scans at the end of Cycle 6 had revealed evidence of colitis in the distal colon. Testing of the patient's stool sample for Clostridium difficile were performed with negative results. Symptoms of colitis and diarrhea were well controlled with concomitant use of lomotil and budesonide. For the following 5 cycles, the patient experienced no new adverse events and reportedly even began to exercise again. However, near the end of Cycle 12 the patient reported to their local emergency department with again symptoms of diarrhea. A CT scan again showed evidence of colitis and the patient began treatment with prednisone for 16 days while study treatment was held. A colonoscopy was performed and Clostridium difficile tests were negative again. However, further work up for colitis reported the presence of Campylobacter jejuni in the stool culture consistent with active colitis. Study treatment was then restarted at the $3^{\text {rd }}$ and final dose reduction level of $30 \mathrm{mg} 4 / 3$. Following resolution of the colitis event, the patient reported minimal occurrences of diarrhea that appears to have been well controlled by loperamide.

No new adverse events or dose interruptions were reported after Cycle 13 until Cycle 27 when the patient experienced episodes of Grade 1 bronchitis and productive cough and study treatment was held for 11 days. A chest X-ray showed stable to moderate right pleural effusion and vague retrocardiac streaky densities, possibly reflecting atelectasis aspiration or infection. A CT scan of the chest ruled out pneumonitis and thoracentesis was not performed due to the apparent lack of fluid present to drain. The patient continued to have a mild cough that was reportedly much improved and the bronchitis resolved within 1 week of starting. Study treatment was restarted again at $30 \mathrm{mg} \mathrm{4/3}$.

Since the end of Cycle 10 and Cycle 26 disease assessments, the patient's non-target segment IV liver and right hilar soft tissue lesions have been absent, respectively, leaving only pleural effusion as the currently present site of disease. The patient has not required any thoracentesis for pleural effusion. The best overall response for every disease assessment by CT scan to date has been a non-complete remission (CR)/non-progressive disease (PD) (Table 1).

As of 07 July 2018, the patient remains on active treatment for over 2 years in Cycle 42.

\section{Discussion}

Overall CUDC-907 treatment has been well tolerated with adverse events consisting primarily of mild-moderate diarrhea and intermittent thrombocytopenia. Both of these events are commonly associated with CUDC-907 treatment as well as with other HDAC and PI3K inhibitors [16] [17]. Diarrhea events were generally managed by the use of loperamide or lomotil and budesonide. The Grade 1 colitis event reported in Cycle 12 was considered related to the presence of 
Table 1. Summary of Patient Disease Assessments.

\begin{tabular}{ccccc}
\hline \multirow{2}{*}{ Assessment } & \multicolumn{3}{c}{ Non-Target Sites of Disease } & \\
\cline { 2 - 4 } & Segment IV liver & $\begin{array}{c}\text { Right hilar soft } \\
\text { tissue }\end{array}$ & Pleural effusion & Best Overall Response \\
\hline Cycle 2 & Present & Present & Present & Non-CR/non-PD \\
Cycle 4 & Present & Present & Present & Non-CR/non-PD \\
Cycle 6 & Present & Present & Present & Non-CR/non-PD \\
Cycle 8 & Present & Present & Present & Non-CR/non-PD \\
Cycle 10 & Absent & Present & Present & Non-CR/non-PD \\
Cycle 14 & Absent & Present & Present & Non-CR/non-PD \\
Cycle 18 & Absent & Present & Present & Non-CR/non-PD \\
Cycle 22 & Absent & Present & Present & Non-CR/non-PD \\
Cycle 26 & Absent & Absent & Present & Non-CR/non-PD \\
Cycle 30 & Absent & Absent & Present & Non-CR/non-PD \\
Cycle 34 & Absent & Absent & Present & Non-CR/non-PD \\
Cycle 38 & Absent & Absent & Present & Non-CR/non-PD \\
\hline Disease assessments by CT scan. Present refers to continued presence without of equivocal progression.
\end{tabular}

Campylobacter jejuni and not study treatment. This was discovered following additional work up due to the patient's daughter also reporting events of diarrhea and may be associated with environmental factors. Furthermore, the patient also has a remote history of colitis which may have been related to prior exposure to Campylobacter jejuni. Thrombocytopenia events were managed by the use of dose holds and/or reductions.

The patient has reported encouragingly prolonged and ongoing disease stabilization for over 32 months. Due to the patient not having target lesions to measure, the direct quantitative impact of CUDC-907 on anti-tumor activity cannot be ascertained in this case. However, by the end of Cycles 10 and 26, the patient's segment IV liver and right hilar soft tissue lesions were absent, respectively (Table 1). In addition, thoracentesis has not been required on study, suggesting prolonged stability of the patient's pleural effusion since treatment was initiated. Hence, the patient's assessable sites of disease either gradually receded or remained stable through CUDC-907 treatment, demonstrating a marked improvement in disease control compared to their previous 2 therapies. Importantly the patient has demonstrated signs of improved quality of life since beginning treatment, such as the ability to exercise again.

The median survival time for NMC patients from diagnosis is reported to be less than 7 months, and there is a greater than $80 \%$ likelihood of death within the first year of diagnosis for adult patients [1] [18] [19]. So far as current literature suggests, there appears only two cases of NMC patients reporting longer survival times than the patient presented here. In one case, a 16-year old boy with an endobronchial lesion (NUT variant) underwent resection and 4 cycles of 
adjuvant cisplatin and docetaxel followed by radiation therapy, was reported to be disease free 34 months after diagnosis [20]. In the other case, a 10-year old boy with a tumor in the iliac bone (BRD4-NUT positive) underwent combined modality therapy used for inoperable Ewing sarcoma, consisting of two courses of vincristine, doxorubicin, and ifosfamide, alternating with one course of cisplatin, doxorubicin, and ifosfamide for 35 weeks. During the first 2 cycles the patient also underwent hyper-fractionated accelerated radiotherapy to the tumor area. A post-treatment biopsy revealed no viable tumor cells and the patient was reportedly in continued complete remission 13 years later [21].

Presently there is no standard of care treatment in NMC due to the rarity and fulminant nature of the disease [18]. BET inhibitors have been shown to competitively inhibit BRD-NUT chromatin interactions, down regulate $M Y C$ and $M Y C$-dependent gene expression, and promote cellular differentiation [22]. Objective responses in NMC have been reported from treatment with BET inhibitors OTX015/MK-8628 and GSK525762 [23] [24]. However, the effectiveness of this drug class is unclear due to a lack of clinical data and restored MYC expression through WNT signaling has been proposed as a mechanism for acquired resistance to BET inhibitors [24] [25] [26]. BET inhibition was unfortunately not a successful approach in this patient who progressed less than 3 months after treatment initiation. Another potentially promising approach in NMC has been the investigation of HDAC inhibitors (HDACi). NMC is hypothesized to be driven by an imbalance of histone acetylation/deacetylation leading to the arrest of differentiation and dysregulation of oncogenic MYC activity. Blocking endogenous histone deacetylation is believed to restore this imbalance and increase the expression of pro-differentiation genes [27]. Objective responses from the use of HDACi vorinostat have been reported in at least 2 NMC cases, demonstrating the potential effectiveness of such treatments [28] [29].

CUDC-907 potently inhibits HDAC and PI3K activities, both of which have been shown to individually suppress MYC-induced oncogenic transcriptional programs via different mechanisms. HDAC inhibition potently suppresses expression of MYC at the transcriptional level, while PI3K inhibition results in enhanced ubiquitin-mediated MYC protein degradation at the post-translational level. When combined, there is preclinical evidence that dual HDAC and PI3K inhibition results in synergistic suppression of MYC and anti-cancer effects in multiple MYC-driven models, including BRD4-NUT NMC [15] [30] [31] [32] [33]. CUDC-907 has also demonstrated promising and durable clinical activity in MYC-altered diffuse large B-cell lymphoma (DLBCL) patients [16]. This case highlights the general tolerability and promising activity of CUDC-907 in a tumor type that is rapidly fatal and which has few therapeutic options available. The study CUDC-907-102 continues to explore whether CUDC-907 may be an effective treatment option for more patients with this devastating disease.

\section{Conclusion}

NMC is a rare, but underdiagnosed, rapidly fatal disease for which there are no 
established effective treatment options available. The dysregulation of MYC is believed to be a central driver in the pathogenesis of NMC [13] [14]. CUDC-907, a dual HDAC and PI3K inhibitor, has demonstrated preclinical suppression of MYC expression and protein stability, potent anti-cancer activity in multiple MYC-driven tumor models including NMC, and promising clinical activity in MYC-altered DLBCL [15] [16]. In this case, a 61-year old female NMC patient who had previously progressed rapidly through treatment with platinum-based chemoradiotherapy and an investigational BET inhibitor has reported prolonged disease stabilization (ongoing at over 32 months) and manageable toxicities from treatment with CUDC-907. This case demonstrates a rare success in the treatment of a particularly devastating disease using only a novel oral small molecule inhibitor, warranting further investigation.

\section{Conflicts of Interest}

The authors declare no conflicts of interest regarding the publication of this paper.

\section{References}

[1] Bauer, D.E., et al. (2012) Clinicopathologic Features and Long-Term Outcomes of NUT Midline Carcinoma. Clinical Cancer Research, 18, 5773-5779. https://doi.org/10.1158/1078-0432.CCR-12-1153

[2] D'Ambrosio, L., et al. (2017) Alpha-Fetoprotein Elevation in NUT Midline Carcinoma: A Case Report. BMC Cancer, 17, 266. https://doi.org/10.1186/s12885-017-3262-0

[3] French, C. (2014) NUT Midline Carcinoma. Nature Reviews Cancer, 14, 149-150. https://doi.org/10.1038/nrc3659

[4] French, C.A., et al. (2014) NSD3-NUT Fusion Oncoprotein in NUT Midline Carcinoma: Implications for a Novel Oncogenic Mechanism. Cancer Discovery, 4, 928-941. https://doi.org/10.1158/2159-8290.CD-14-0014

[5] Haack, H., et al. (2009) Diagnosis of NUT Midline Carcinoma Using a NUT-Specific Monoclonal Antibody. The American Journal of Surgical Pathology, 33, 984-991. https://doi.org/10.1097/PAS.0b013e318198d666

[6] Chau, N.G., et al. (2016) Intensive Treatment and Survival Outcomes in NUT Midline Carcinoma of the Head and Neck. Cancer, 122, 3632-3640. https://doi.org/10.1002/cncr.30242

[7] Muller, S., Filippakopoulos, P. and Knapp, S. (2011) Bromodomains as Therapeutic Targets. Expert Reviews in Molecular Medicine, 13, e29. https://doi.org/10.1017/S1462399411001992

[8] Devaiah, B.N., et al. (2016) BRD4 Is a Histone Acetyltransferase that Evicts Nucleosomes from Chromatin. Nature Structural \& Molecular Biology, 23, 540-548. https://doi.org/10.1038/nsmb.3228

[9] French, C.A., Miyoshi, I., Kubonishi, I., Grier, H.E., Perez-Atayde, A.R. and Fletcher, J.A. (2003) BRD4-NUT Fusion Oncogene: A Novel Mechanism in Aggressive Carcinoma. Cancer Research, 63, 304-307.

[10] Kuroda, S., et al. (2015) Cytological Features of a Variant NUT Midline Carcinoma of the Lung Harboring the NSD3-NUT Fusion Gene: A Case Report and Literature 
Review. Case Reports in Pathology, 2015, 572951. https://doi.org/10.1155/2015/572951

[11] Mills, A.F., et al. (2014) NUT Midline Carcinoma: A Case Report with a Novel Translocation and Review of the Literature. Head and Neck Pathology, 8, 182-186. https://doi.org/10.1007/s12105-013-0479-3

[12] Shatavi, S., Fawole, A., Haberichter, K., Jaiyesimi, I. and French, C. (2016) Nuclear Protein in Testis (NUT) Midline Carcinoma with a Novel Three-Way Translocation (4;15;19)(q13;q14;p13.1). Pathology (Phila.), 48, 620-623. https://doi.org/10.1016/j.pathol.2016.06.010

[13] Alekseyenko, A.A., et al. (2015) The Oncogenic BRD4-NUT Chromatin Regulator Drives Aberrant Transcription within Large Topological Domains. Genes \& Development, 29, 1507-1523. https://doi.org/10.1101/gad.267583.115

[14] Grayson, A.R., et al. (2014) MYC, a Downstream Target of BRD-NUT, Is Necessary and Sufficient for the Blockade of Differentiation in NUT Midline Carcinoma. Oncogene, 33, 1736-1742. https://doi.org/10.1038/onc.2013.126

[15] Sun, K., et al. (2017) Dual HDAC and PI3K Inhibitor CUDC-907 Downregulates MYC and Suppresses Growth of MYC-Dependent Cancers. Molecular Cancer Therapeutics, 16, 285-299. https://doi.org/10.1158/1535-7163.MCT-16-0390

[16] Oki, Y., et al. (2017) CUDC-907 in Relapsed/Refractory Diffuse Large B-Cell Lymphoma, Including Patients with MYC-Alterations: Results from an Expanded Phase I Trial. Haematologica, 102, 1923-1930. https://doi.org/10.3324/haematol.2017.172882

[17] Younes, A., et al. (2016) Safety, Tolerability, and Preliminary Activity of CUDC-907, a First-in-Class, Oral, Dual Inhibitor of HDAC and PI3K, in Patients with Relapsed or Refractory Lymphoma or Multiple Myeloma: An Open-Label, Dose-Escalation, Phase 1 Trial. The Lancet Oncology, 17, 622-631. https://doi.org/10.1016/S1470-2045(15)00584-7

[18] Cao, J., Chen, D., Yang, F., Yao, J., Zhu, W. and Zhao, C. (2017) NUT Midline Carcinoma as a Primary Lung Tumor: A Case Report. Journal of Thoracic Disease, 9, E1045-E1049. https://doi.org/10.21037/jtd.2017.11.50

[19] French, C.A. (2010) Demystified Molecular Pathology of NUT Midline Carcinomas. Journal of Clinical Pathology, 63, 492-496. https://doi.org/10.1136/jcp.2007.052902

[20] Fujioka, N., French, C.A., Cameron, M.J. and Kratzke, R.A. (2010) Long-Term Survival of a Patient with Squamous Cell Carcinoma Harboring NUT Gene Rearrangement. Journal of Thoracic Oncology, 5, 1704-1705. https://doi.org/10.1097/JTO.0b013e3181ebaa20

[21] Mertens, F., Wiebe, T., Adlercreutz, C., Mandahl, N. and French, C.A. (2007) Successful Treatment of a Child with $\mathrm{t}(15 ; 19)$-Positive Tumor. Pediatric Blood \& Cancer, 49, 1015-1017. https://doi.org/10.1002/pbc.20755

[22] Delmore, J.E., et al. (2011) BET Bromodomain Inhibition as a Therapeutic Strategy to Target c-Myc. Cell, 146, 904-917. https://doi.org/10.1016/j.cell.2011.08.017

[23] O’Dwyer, P.J., et al. (2016) Abstract CT014: GSK525762, a Selective Bromodomain (BRD) and Extra Terminal Protein (BET) Inhibitor: Results from Part 1 of a Phase I/II Open-Label Single-Agent Study in Patients with NUT Midline Carcinoma (NMC) and Other Cancers. Cancer Research, 76, CT014. https://doi.org/10.1158/1538-7445.AM2016-CT014

[24] Stathis, A., et al. (2016) Clinical Response of Carcinomas Harboring the BRD4-NUT Oncoprotein to the Targeted Bromodomain Inhibitor OTX015/MK-8628. Cancer 
Discovery, 6, 492-500.

https://doi.org/10.1158/2159-8290.CD-15-1335

[25] Fong, C.Y., et al. (2015) BET Inhibitor Resistance Emerges from Leukaemia Stem Cells. Nature, 525, 538-542. https://doi.org/10.1038/nature14888

[26] Rathert, P., et al. (2015) Transcriptional Plasticity Promotes Primary and Acquired Resistance to BET Inhibition. Nature, 525, 543-547. https://doi.org/10.1038/nature14898

[27] Schwartz, B.E., et al. (2011) Differentiation of NUT Midline Carcinoma by Epigenomic Reprogramming. Cancer Research, 71, 2686-2696. https://doi.org/10.1158/0008-5472.CAN-10-3513

[28] Ball, A., Bromley, A., Glaze, S., French, C.A., Ghatage, P. and Köbel, M. (2012) A Rare Case of NUT Midline Carcinoma. Gynecologic Oncology Case Reports, 3, 1-3. https://doi.org/10.1016/j.gynor.2012.09.004

[29] Maher, O.M., Christensen, A.M., Yedururi, S., Bell, D. and Tarek, N. (2015) Histone Deacetylase Inhibitor for NUT Midline Carcinoma. Pediatric Blood \& Cancer, 62 , 715-717. https://doi.org/10.1002/pbc.25350

[30] Pei, Y., et al. (2016) HDAC and PI3K Antagonists Cooperate to Inhibit Growth of MYC-Driven Medulloblastoma. Cancer Cell, 29, 311-323. https://doi.org/10.1016/j.ccell.2016.02.011

[31] Qian, C., et al. (2012) Cancer Network Disruption by a Single Molecule Inhibitor Targeting both Histone Deacetylase Activity and Phosphatidylinositol 3-Kinase Signaling. Clinical Cancer Research, 18, 4104-4113. https://doi.org/10.1158/1078-0432.CCR-12-0055

[32] Rahmani, M., Aust, M.M., Benson, E.C., Wallace, L., Friedberg, J. and Grant, S. (2014) PI3K/mTOR Inhibition Markedly Potentiates HDAC Inhibitor Activity in NHL Cells through BIM- and MCL-1-Dependent Mechanisms in Vitro and in Vivo. Clinical Cancer Research, 20, 4849-4860. https://doi.org/10.1158/1078-0432.CCR-14-0034

[33] Sander, S., et al. (2012) Synergy between PI3K Signaling and MYC in Burkitt Lymphomagenesis. Cancer Cell, 22, 167-179. https://doi.org/10.1016/j.ccr.2012.06.012 